\title{
How does exercise treatment compare with antihypertensive medications? A network meta-analysis of 391 randomised controlled trials assessing exercise and medication effects on systolic blood pressure
}

\author{
Huseyin Naci, ${ }^{1}$ Maximilian Salcher-Konrad, ${ }^{1}$ Sofia Dias, ${ }^{02,3}$ Manuel R Blum, ${ }^{4,5,6}$ \\ Samali Anova Sahoo, ${ }^{7}$ David Nunan, ${ }^{8}$ John P A Ioannidis ${ }^{5,6,9}$
}

For numbered affiliations see end of article.

\section{Correspondence to}

Dr Huseyin Naci, Department of Health Policy, London School of Economics and Political Science, London WC2A 2AE, UK ; H.Naci@|se.ac.uk

Accepted 29 October 2018 Published Online First 18 December 2018

\section{Linked}

http://dx.doi.org/10.1136/ bjsports-2018-100359

- http://dx.doi.org/10.1136/ bjsports-2019-100892

Check for updates

(C) Author(s) (or their employer(s)) 2019. No commercial re-use. See rights and permissions. Published by BMJ.

To cite: Naci H, Salcher-

Konrad M, Dias S,

et al. Br J Sports Med

2019:53:859-869.

\section{ABSTRACT}

Objective To compare the effect of exercise regimens and medications on systolic blood pressure (SBP).

Data sources Medline (via PubMed) and the Cochrane Library.

Eligibility criteria Randomised controlled trials (RCTs) of angiotensin-converting enzyme inhibitors (ACE-I), angiotensin-2 receptor blockers (ARBs), $\beta$-blockers, calcium channel blockers (CCBs) and diuretics were identified from existing Cochrane reviews. A previously published meta-analysis of exercise interventions was updated to identify recent RCTs that tested the SBP-lowering effects of endurance, dynamic resistance, isometric resistance, and combined endurance and resistance exercise interventions (up to September 2018).

Design Random-effects network meta-analysis. Outcome Difference in mean change from baseline SBP between comparator treatments (change from baseline in one group minus that in the other group) and its 95\% credible interval $(95 \% \mathrm{Crl})$, measured in $\mathrm{mmHg}$.

Results We included a total of 391 RCTs, 197 of which evaluated exercise interventions (10 461 participants) and 194 evaluated antihypertensive medications (29 281 participants). No RCTs compared directly exercise against medications. While all medication trials included hypertensive populations, only 56 exercise trials included hypertensive participants ( $\geq 140 \mathrm{mmHg}$ ), corresponding to 3508 individuals. In a 10\% random sample, risk of bias was higher in exercise RCTs, primarily due to lack of blinding and incomplete outcome data. In analyses that combined all populations, antihypertensive medications achieved higher reductions in baseline SBP compared with exercise interventions (mean difference $-3.96 \mathrm{mmHg}, 95 \% \mathrm{Crl}-5.02$ to -2.91 ). Compared with control, all types of exercise (including combination of endurance and resistance) and all classes of antihypertensive medications were effective in lowering baseline SBP. Among hypertensive populations, there were no detectable differences in the SBP-lowering effects of $A C E-I, A R B, \beta$-blocker and diuretic medications when compared with endurance or dynamic resistance exercise. There was no detectable inconsistency between direct and indirect comparisons. Although there was evidence of small-study effects, this affected both medication and exercise trials.

Conclusions The effect of exercise interventions on SBP remains under-studied, especially among hypertensive populations. Our findings confirm modest but consistent reductions in SBP in many studied exercise interventions

\section{What is already known?}

- Exercise interventions are effective in lowering systolic blood pressure.

What are the new findings?

Across all populations, individuals who receive antihypertensive medications tend to achieve greater reductions in systolic blood pressure than those who adopt structured exercise regimens.

- In populations with hypertension, different types of exercise interventions appear to be as equally effective as most antihypertensive medications.

- Structured exercise has not been evaluated as extensively as antihypertensive medications.

across all populations but individuals receiving medications generally achieved greater reductions than those following structured exercise regimens. Assuming equally reliable estimates, the SBP-lowering effect of exercise among hypertensive populations appears similar to that of commonly used antihypertensive medications. Generalisability of these findings to real-world clinical settings should be further evaluated.

\section{INTRODUCTION}

High systolic blood pressure (SBP) is a major modifiable risk factor for cardiovascular disease. ${ }^{1}$ Individuals with high SBP are at elevated risk of cardiovascular disease and death ${ }^{2-4}$ and high SBP is the leading cause of death and disability around the world. ${ }^{5}$ Over the past half century, several classes of pharmacological treatment options have received approval to be prescribed for blood pressure-lowering. ${ }^{6}$ The mortality and morbidity benefits of these antihypertensive medication options have been extensively documented in randomised controlled trials (RCTs) and meta-analyses. ${ }^{78}$

As the burden of cardiovascular disease continues to rise, ${ }^{9}$ the use of medications targeting high blood pressure is sharply increasing. ${ }^{10}$ In England, the number of adults taking blood pressure-lowering medications increased by approximately $50 \%$ 
from 2006 to $2016 .{ }^{11}$ This upward trend will likely increase, as recent changes to major clinical practice guidelines developed by prominent organisations such as the American Heart Association (AHA) and the American College of Cardiology (ACC) have lowered the SBP threshold for the definition of hypertension. ${ }^{12}$ These changes are expected to increase the number of people labelled as having hypertension and treated with medications. ${ }^{1314}$

Such an increase may lead to inadvertent adverse events at the population level, as the number of people taking multiple medications continues to rise ${ }^{15}$; polypharmacy represents a major risk factor for drug-related morbidity and mortality. ${ }^{16}$ Prescription drugs also contribute to rising healthcare expenditures. Spending on medications accounts for about $18 \%$ of total health spending on average across European countries. ${ }^{17}$ Recent increases in medication-related costs have prompted significant policy and clinical attention to the comparative effectiveness of new and existing medications. ${ }^{18}$ Meanwhile, relatively little attention has been given to promoting the wider adoption of non-pharmacological interventions such as exercise.

Exercise interventions have indisputable benefits for cardiovascular disease and beyond. ${ }^{19} 20$ According to a pooled analysis of observational cohort studies, men and women with high levels of leisure time physical activity had a $24 \%$ and $27 \%$ lower risk of cardiovascular disease, respectively, than men and women with low levels of physical activity. ${ }^{21}$ In addition, previous meta-analyses of RCTs showed that exercise is effective in improving established cardiovascular risk factors: exercise interventions reduce waist circumference, ${ }^{22}$ improve glycated haemoglobin (HbA1c), ${ }^{23}$ lower serum triglycerides ${ }^{24}$ and increase high-density lipoprotein. ${ }^{25}$

Exercise also has well-documented benefits in lowering SBP. ${ }^{26}$ In a previous meta-analysis of 93 RCTs conducted among 5223 healthy adults, SBP was reduced after endurance, dynamic resistance and isometric resistance exercise regimens. ${ }^{27}$ Although recent AHA/ACC guidelines emphasise the role of lifestyle interventions, including exercise, in the management and treatment of hypertension, they consider pharmacological and non-pharmacological interventions in isolation. ${ }^{12}$ It would be very important to evaluate the comparative SBP-lowering effects of exercise and medication interventions.

In a previous meta-epidemiological study, we evaluated the comparative effectiveness of pharmacological and non-pharmacological interventions on mortality. ${ }^{28}$ We found structured exercise interventions to be as equally effective as several frequently used medications in terms of their mortality benefits in the secondary prevention of coronary heart disease, rehabilitation after stroke, treatment of heart failure and prevention of diabetes. However, the amount of evidence on the mortality benefits of exercise was considerably smaller than that on medications. In addition, there was a paucity of available information on the 'formulation' and 'dose' of different types of exercise interventions, and also on the characteristics of people that stood to benefit from such interventions.

In this study, we set out to perform a network meta-analysis to compare systematically the SBP-lowering effects of exercise and medications. Our objective was to evaluate how different types and intensities of exercise fared against different classes and doses of antihypertensive medications in terms of lowering baseline SBP levels. In addition, we assessed the comparative SBP-lowering effects of exercise and medications specifically among hypertensive populations.

\section{METHODS}

\section{Identification of available evidence}

As previously, ${ }^{28}$ we identified the relevant body of evidence in three steps. First, one researcher (HN) searched Medline via PubMed for the most recently published comprehensive meta-analyses of RCTs evaluating the effectiveness of exercise interventions on lowering SBP (see search strategy in online supplementary appendix 1).

Second, one researcher (HN up to August 2017 and MSK from August 2017 to September 2018) searched Medline via PubMed to identify recently published RCTs of exercise interventions aimed at lowering SBP that were published after the end date of electronic database search in the meta-analyses identified in step one (see search strategy in online supplementary appendix 2). Accordingly, our search covered the period from February 2012 to September 2018. Two researchers (SAS and HN, up to August 2017) and one researcher (MSK, from August 2017 to September 2018) screened identified titles and abstracts according to prespecified eligibility criteria. Participants of interest included adults (with or without hypertension) with no cardiovascular disease, cerebrovascular disease, diabetes or other chronic conditions such as cancer. Eligible interventions were any form of structured exercise of any frequency, duration or intensity. Eligible comparator interventions included usual practice (no exercise), other exercise regimens, or medications. Studies were included if they lasted at least 4 weeks and reported SBP at baseline and follow-up (or change from baseline) for intervention and comparator arms or the difference in means between the two arms. One researcher (MB) contacted the corresponding authors of recently published RCTs to obtain missing outcome data in the papers. Following title and abstract screening, three researchers (MB, MSK and $\mathrm{HN}$ ) reviewed potentially relevant full text articles to determine study eligibility. Disagreements were resolved by consensus.

Third, one researcher (MSK) searched the Cochrane Library to identify published meta-analyses of RCTs of prescription medications aimed at lowering SBP with similar participant populations to those in the meta-analyses of exercise trials (ie, adults in whom the blood pressure lowering effect of an intervention can be observed, excluding individuals with other conditions potentially causing hypertension, such as renal failure). Comparators in eligible medication trials included placebo, other medications, doses, or usual care. The list of relevant medication classes was identified using the clinical practice guidelines developed by the National Institute for Health and Care Excellence (NICE) ${ }^{29}$ and the European Society of Hypertension/European Society of Cardiology (ESH/ESC). ${ }^{30}$ We also used the British National Formulary (BNF) to determine the eligible doses of individual antihypertensive medications. ${ }^{31}$ Only trial arms of RCTs of medications from guideline-recommended medication classes and BNF-approved doses were eligible for inclusion in our review. We did not run additional searches to update the list of medication RCTs included in previous meta-analyses, since they were deemed to be sufficiently up-to-date and, in contrast to exercise trials, the amount of evidence for medication trials was already very large.

\section{Data extraction}

We adopted a two-tiered data extraction strategy. For eligible RCTs of medications, we relied on the information reported in the published Cochrane meta-analyses. We divided the sample of RCTs and two researchers (MSK and MB) extracted information on author name, trial reference, publication year, interventions 
(including dose), comparators, sample size (total number of randomly assigned participants or total number of participants with outcome measurement) per trial arm, and outcome data.

For eligible RCTs of exercise interventions, we carried out primary data collection from each publication. In addition to the data items captured from medication RCTs, we collected detailed information on the characteristics of participants (eg, mean age, proportion female) and interventions (type, intensity, frequency, duration). In terms of outcome data, we focused on SBP, as it has been consistently associated with cardiovascular risk in epidemiological and experimental studies. ${ }^{12}$ SBP is also more commonly reported than diastolic blood pressure. ${ }^{27} \mathrm{We}$ set out to extract the mean change from baseline SBP levels and its standard deviation (SD) in each trial arm. When the mean change from baseline was not available, we obtained the mean and SD of SBP levels at baseline and follow-up in each arm and thus calculated the mean change from baseline for each study.

Data on the SD of change from baseline SBP were rarely available. We therefore relied on standard errors, 95\% confidence intervals, $\mathrm{P}$ values or $\mathrm{t}$ statistics to calculate $\mathrm{SD}$, as recommended by the Cochrane Handbook. ${ }^{32}$ When no information was available to calculate SD, we imputed missing values by using a correlation coefficient of 0.8 between baseline and follow-up SBP. We tested the sensitivity of our findings to different correlation coefficients and confirmed the consistency of results across different sets of analyses (see online supplementary appendix 4). Two researchers extracted outcome data (SAS and MB up to August 2017, and HN and MSK from August 2017 to September 2018) and another researcher independently appraised the accuracy of the information.

\section{Categorisation of available evidence}

Exercise was defined as a subset of physical activity that is structured and repetitive with the objective of improving or maintaining physical fitness. ${ }^{33}$ We divided exercise interventions into four major categories: (1) endurance, (2) dynamic resistance, (3) isometric resistance, and (4) a combination of endurance and dynamic resistance. ${ }^{27}$ Endurance exercise included interventions aimed at increasing heart rate and energy expenditure. Examples of endurance exercise included walking, jogging, running, cycling and swimming. Interval training was considered as endurance exercise. We labelled exercise interventions as resistance training if they were aimed at increasing muscular strength and power. Strength training with dumbbells was a typical form of resistance exercise. We categorised exercise interventions as isometric exercise if they involved sustained contraction against an immovable load.

Intensity of exercise interventions was categorised into low, moderate and high using the classification developed by the American College of Sports Medicine. ${ }^{34}$ The majority of exercise RCTs reported relevant information such as percent of heart rate reserve (\% HRR), percent of maximal heart rate $\left(\% \mathrm{HR}_{\max }\right)$, percent of maximal oxygen uptake $\left(\% \mathrm{VO}_{2 \max }\right)$, or percent of one repetition maximum ( $\% 1 \mathrm{RM})$ to categorise the relative or absolute intensity of exercise interventions. In cases where such information was not available, we relied on the study authors' reporting to determine the intensity of physical activity.

Individual medications were categorised into the following antihypertensive medication classes: angiotensin-converting enzyme inhibitors (ACE-I), angiotensin-2 receptor blockers (ARBs), $\beta$-blockers, calcium channel blockers (CCBs) and diuretics. Medications were also divided into low and high doses according to the BNF, assigning them to 'low' if at or below the mid-point of recommended doses in the BNF and 'high' if above the mid-point of recommended doses.

We categorised exercise trials according to the study-level mean baseline SBP of the participant population. While the RCTs of antihypertensive medications included only hypertensive participants (with baseline SBP $\geq 140 \mathrm{mmHg}$ ), exercise trials had more variable inclusion criteria. In our primary analysis, participant populations were labelled as 'hypertensive' if exercise trials included adults with mean baseline SBP of at least $140 \mathrm{mmHg}$, which was consistent with the original definition of hypertension until the changes introduced by the 2017 AHA/ ACC guidelines.

We also considered additional cut-offs to define hypertension in two sensitivity analyses. In the first set of sensitivity analyses, we labelled populations in exercise RCTs as hypertensive if they had an average SBP of at least $130 \mathrm{mmHg}$, which corresponds to the new blood pressure threshold to define hypertension in the 2017 AHA/ACC guidelines. ${ }^{12}$ In the second set of sensitivity analyses, we tested a cut-off of $150 \mathrm{mmHg}$ for mean SBP in exercise trials, as this more closely matched the mean SBP of the trial populations in medication trials.

\section{Risk of bias assessment}

We used the Cochrane risk of bias tool to evaluate the internal validity of results in a $10 \%$ random sample of medication $(n=20)$ and exercise RCTs $(\mathrm{n}=20)$ (online supplementary appendix $5) .{ }^{35}$ Two researchers ( $\mathrm{HN}$ and $\mathrm{MB}$ ) reviewed the publications of selected trials to determine whether the investigators used appropriate methods to (1) generate a random allocation sequence (selection bias), (2) conceal the sequence of treatment allocation from trial investigators and participants before the trial (selection bias), (3) mask participants and investigators from knowledge of treatment allocation during the trial (performance bias and detection bias), and (4) deal with missing outcome data (attrition bias). We consistently rated the selective outcome reporting domain as 'unclear', as there was inadequate information available in the trials to evaluate planned versus reported outcomes.

\section{Statistical analysis}

We first qualitatively synthesised included trials and described the types of direct and indirect comparisons and their relative contributions to the overall body of available evidence.

We then developed network diagrams to visualise the relative amount of available evidence on exercise and medications. ${ }^{36}$ Nodes represented different exercise and medication interventions and lines connecting the nodes represented the direct head-to-head comparisons between interventions. In network diagrams, the size of each node and the thickness of each line connecting the nodes were proportional to the number of participants. All network diagrams were generated using Stata version $15 .^{37}$

To estimate the comparative effectiveness of exercise and medications on SBP-lowering, we performed network meta-analyses. ${ }^{38}$ Such analyses allow for the comparison of treatments that have not been directly compared with each other in headto-head studies. ${ }^{39}$ They can also combine evidence obtained from direct and indirect comparisons, thereby improving the precision of treatment effect estimates. ${ }^{404}$ Similar to pair-wise meta-analyses, network meta-analyses preserve the random allocation of participants to different arms within each trial; however, they compare multiple interventions by combining all 
available comparisons between treatments across trials, utilising the totality of the available evidence. ${ }^{42}$

Study-level treatment effects were combined using Bayesian Markov chain Monte Carlo methods in WinBUGS version 1.4.3. ${ }^{43}$ We used the model developed by Dias and colleagues for the NICE Decision Support Unit. ${ }^{44-46}$ Our base-case model assumed that the mean change from baseline in SBP per trial arm had a normal distribution. The relative effects across trials making different comparisons were linked using the identity function. This model took into account the correlations between treatment effects within multi-arm trials.

We used a random-effects model to perform the network meta-analyses, allowing for between-study heterogeneity. ${ }^{47}$ Our models therefore assumed that trial-specific treatment effects were drawn from a normal distribution, with a mean that was specific for each treatment comparison, and a common variance that was shared by all comparisons. We reported the mean treatment effect with $95 \%$ credible intervals $(95 \% \mathrm{CrI})$ of every intervention relative to control and other interventions and the estimated between-study heterogeneity SD with its 95\% CrI.

To test the consistency assumption of the network meta-analysis, we compared the fit of the base-case model to that of an inconsistency model. ${ }^{48} 49$ The latter model did not assume consistency between direct and indirect evidence and instead estimated independent mean treatment effects. ${ }^{50}$ We also examined each data point's contribution to the residual deviance and compared the estimated between-study heterogeneity in each model. We assessed any improvements in fit or reductions in between-study heterogeneity in the inconsistency model, which would suggest potential inconsistency (see online supplementary appendix 6). We plotted the findings of this secondary analysis side-by-side with our base-case model that assumed consistency to compare the results of the two models. We visually inspected the findings and assessed for systematic differences from those obtained from our primary analyses (see online supplementary appendix 6).
We compared the SBP-lowering effects of exercise and antihypertensive medications in three sets of analyses: (1) all exercise interventions versus all antihypertensive medications; (2) different types of exercise interventions versus different classes of medications; and (3) different intensities of exercise interventions versus different doses of medications. We then repeated these analyses and compared the antihypertensive RCTs to a subset of exercise trials that only included hypertensive populations.

We evaluated small-study effects by extending the regression-based approach proposed by Moreno and colleagues. ${ }^{51-53}$ We regressed the treatment effects against their standard errors and predicted the pooled effect size for an ideal study of infinite size (ie, with zero SE), assuming that smaller studies would be more biased than larger studies. ${ }^{54}$ This meta-regression allowed for a different mean bias according to type of comparison (ie, mean bias due to small-study effects was assumed to be different for RCTs evaluating the effect of exercise versus control and medications versus control). ${ }^{55}$

We adopted non-informative prior distributions for treatment effects (normal $(0,10000))$ and the between-trial variance (uniform $(0,10)$ ). Our analyses employed a long burn-in period (50000 iterations) and follow-up period (100000 iterations) to allow for convergence. We ran three chains with different sets of initial values. We visually inspected trace plots for key parameters for each analysis to assess convergence in terms of stability.

\section{RESULTS}

\section{Evidence base for medications}

Using the Cochrane Library, we initially identified 14 potentially relevant meta-analyses of medication therapies aimed at lowering baseline SBP (figure 1). Of these, we selected the most comprehensive meta-analyses within each medication class recommended by the NICE and ESH/ESC guidelines as

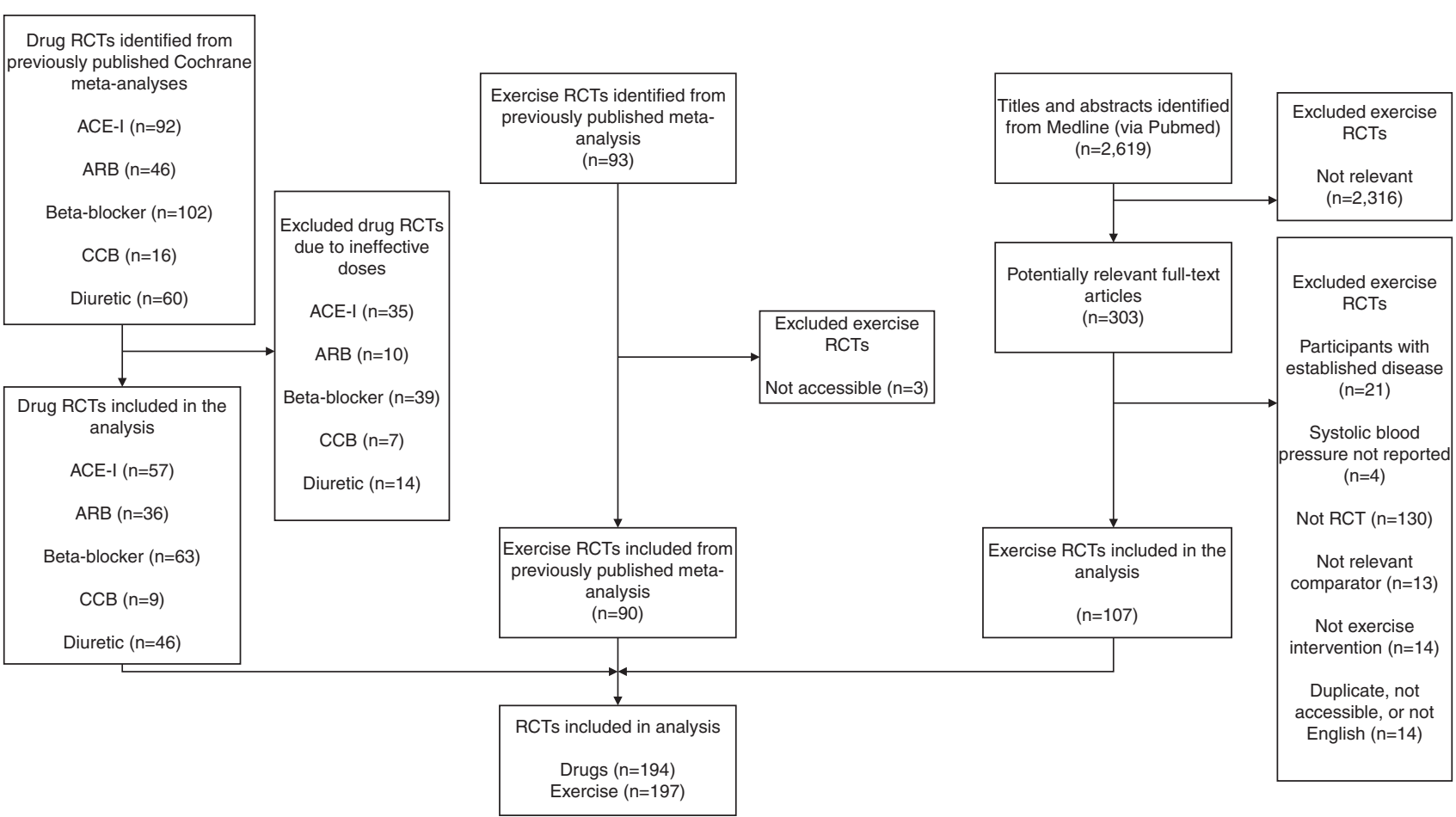

Figure 1 Flow diagram of study identification and selection. ACE-I, angiotensin-converting enzyme inhibitor; ARB, angiotensin-2 receptor blocker; CCB, calcium channel blocker; RCTs, randomised controlled trials. 
first-line therapy for hypertension. ${ }^{56-62}$ In total, these meta-analyses included 316 RCTs. We excluded the trials and trial arms of medications and medication dosages that were not indicated in the BNF. After these exclusions, we included 194 medication RCTs, corresponding to 57 trials of ACE-I, 36 studies of ARBs, 63 studies of $\beta$-blockers, nine studies of CCBs and 46 studies of diuretics. Seventeen RCTs compared one medication class to another.

\section{Evidence base for exercise interventions}

Of 47 potentially relevant reviews of exercise interventions identified from Medline, we considered the meta-analysis conducted by Cornelissen and Smart to be the most comprehensive in terms of its study identification, selection, review and synthesis methods. ${ }^{27}$ This analysis relied on 93 RCTs published up to February 2012. We subsequently updated this review and identified 2619 potentially relevant titles and abstracts published until September 2018 (figure 1). We excluded 2316 records that were irrelevant. Of 303 full-text articles, we included an additional 107 RCTs. In total, we ultimately included 197 RCTs of exercise interventions (see online supplementary appendix 3 for trial characteristics): 115 of these evaluated endurance training interventions including walking, running, cycling or aquatic exercises; 30 RCTs evaluated dynamic resistance interventions; 10 evaluated isometric resistance exercises; and 12 tested endurance and resistance training regimens in combination. The remaining 30 RCTs compared one type of exercise intervention to another. No RCTs compared directly exercise against medications.

\section{Characteristics of exercise and medication RCTs}

RCTs of exercise interventions included substantially fewer participants; average sample size in exercise RCTs was 53 (range 15-464) compared with 139 (7-1092) in RCTs of $\beta$-blockers, 174 (14-625) in studies of ACE-I, 188 (24-2776) for diuretics, 185 (15-397) for CCBs and 292 (40-1369) for ARBs. Mean age ranged from 50.4 for exercise trials to 55.0 for $A R B$ and diuretics trials. On average, a higher proportion of participants were women in RCTs of exercise interventions (61\%) compared with the proportion of women participants in RCTs of medications (ranging from 39\% for ARBs to $47 \%$ for $\beta$-blockers). While the mean SBP at baseline was $132 \mathrm{mmHg}$ for participants in the RCTs of exercise interventions, it was consistently over $150 \mathrm{mmHg}$ in medication RCTs (table 1 ).
Distribution of participants in exercise and medication RCTs In total, 39742 participants were included in RCTs testing the SBP-lowering effects of medications and exercise interventions. While 29281 participants were included in medication trials, 10461 were included in exercise RCTs (figure 2A). On average, trials of individual medication classes had more participants than those included in the RCTs of different types of exercise (figure 2B). The majority of participants included in exercise RCTs were in trials evaluating the effect of endurance training, as compared with control or other exercise interventions $(n=8174)$. Relatively more participants were included in trials evaluating moderate-intensity exercise alone $(n=4675)$ compared with those testing low- and high-intensity interventions (figure 2C). Fifty-six exercise trials included hypertensive participants ( $\geq 140 \mathrm{mmHg}$ ), corresponding to 3508 individuals (figure 3). A total of 6046 and 1828 participants were included in exercise RCTs with hypertensive populations when using a cut-off of $130 \mathrm{mmHg}$ and $150 \mathrm{mmHg}$ for mean baseline SBP, respectively.

\section{Risk of bias}

Figure 4 and online supplementary appendix 5 summarise the risk of bias in a $10 \%$ random sample of exercise and medication RCTs. Seventeen of 20 exercise RCTs were judged to be at high risk of performance and detection bias due to lack of blinding, while only one medication RCT was at high risk of bias on this domain. Risk of attrition bias was also higher in exercise trials $(5 / 20)$ compared with that in medication trials $(0 / 20)$. Inadequate reporting complicated our assessments for selection bias with the majority of both exercise and medication trials rated at unclear risk of bias.

\section{Comparative effects on SBP}

Across all populations, antihypertensive medications (mean difference $-8.80 \mathrm{mmHg}, 95 \% \mathrm{CrI}-9.58$ to -8.02 ) and exercise interventions $(-4.84,95 \% \mathrm{CrI}-5.55$ to -4.13$)$ were both effective in lowering SBP from baseline as compared with control (figure 5A). Populations receiving medications achieved greater reductions in SBP compared with those participating in physical activity interventions $(-3.96,95 \% \mathrm{CrI}-5.02$ to -2.91$)$.

Compared with control, all types of exercise (endurance $-4.88,95 \%$ CrI -5.69 to -4.06 ; resistance -3.50 , 95\% CrI -4.91 to -2.09 ; isometric $-5.65,95 \% \mathrm{CrI}-8.21$ to -3.13 ; and combination of endurance and resistance $-6.49,95 \% \mathrm{CrI}$ -8.17 to -4.82 ) and all classes of antihypertensive medications

Table 1 Overall characteristics of available evidence from randomised controlled trials on exercise interventions and medications

\begin{tabular}{|c|c|c|c|c|c|c|c|c|c|}
\hline & Endurance & $\begin{array}{l}\text { Dynamic } \\
\text { resistance }\end{array}$ & $\begin{array}{l}\text { Isometric } \\
\text { resistance }\end{array}$ & $\begin{array}{l}\text { Combination } \\
\text { exercise* }\end{array}$ & ACE-I & ARB & $\beta$-blocker & CCB & Diuretic \\
\hline Number of trialst & 135 & 48 & 12 & 31 & 57 & 36 & 63 & 9 & 46 \\
\hline Mean age in years & 50.8 & 48.5 & 51.9 & 54.0 & 54.4 & 55.0 & 52.1 & 52.3 & 55.0 \\
\hline Proportion female & $59 \%$ & $60 \%$ & $47 \%$ & $75.4 \%$ & $41 \%$ & $39 \%$ & $47 \%$ & N/A & $43 \%$ \\
\hline $\begin{array}{l}\text { Mean baseline SBP } \\
(\mathrm{mmHg})\end{array}$ & 134 & 125 & 129 & 135 & 157 & 156 & 160 & N/A $\ddagger$ & 158 \\
\hline Mean enrolment (range) & $58(15-464)$ & $35(15-96)$ & $30(15-48)$ & $65(16-387)$ & $174(14-625)$ & $292(40-1369)$ & $139(7-1092)$ & $185(15-397)$ & $188(24-2776)$ \\
\hline Years covered & $1976-2018$ & 1987-2018 & 1992-2018 & 2001-2017 & 1983-2002 & 1995-2004 & $1968-2008$ & 1988-2003 & $1978-2009$ \\
\hline
\end{tabular}

${ }^{*} \mathrm{~N} / \mathrm{A}$ : not sufficient information reported in meta-analysis report and supplementary material.

tNumber of trials does not add up to total number of RCTs included in the analysis, as some RCTs included more than one class of antihypertensive medications or one type of exercise interventions.

¥Combination of endurance exercise and dynamic resistance training.

ACE-I, angiotensin- converting enzyme inhibitor; ARB, angiotensin-2 receptor blocker; CCB, calcium channel blocker; RCTs, randomised controlled trials; SBP, systolic blood pressure. 
A

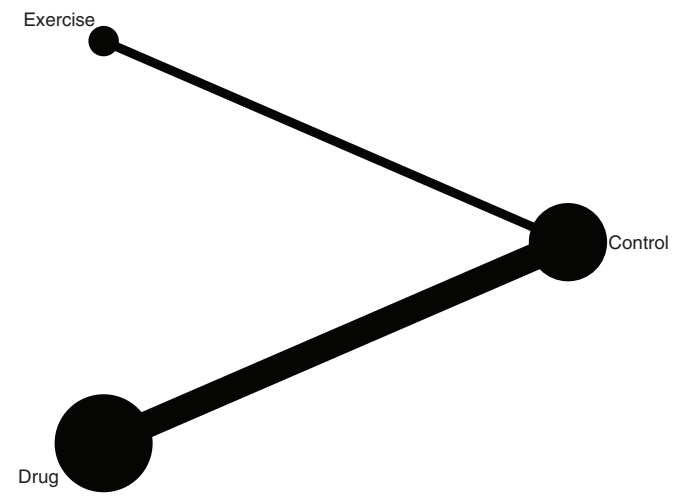

B

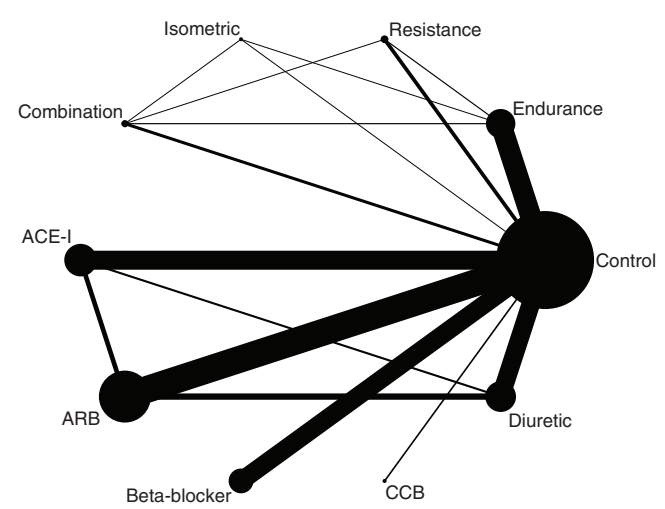

C

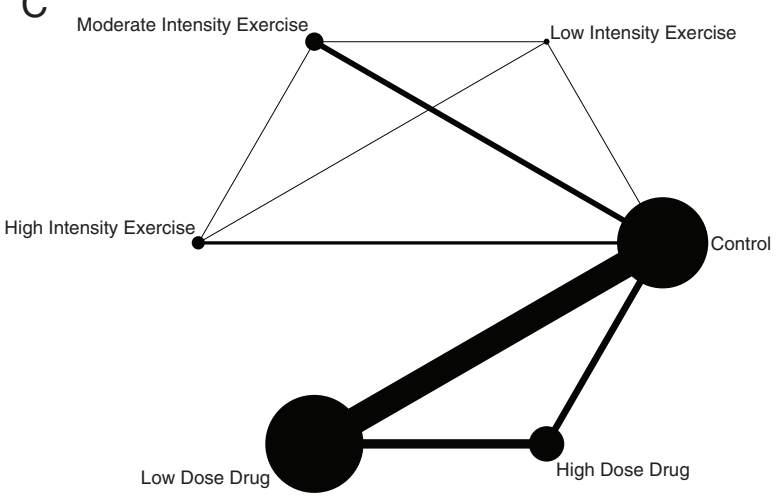

Figure 2 Available evidence comparing (A) exercise versus medications; (B) different types of exercise versus classes of medications; and $(C)$ different intensities of exercise versus doses of medications. The nodes represent different interventions and the lines connecting the nodes represent direct head-to-head randomised controlled trials comparing the interventions. The size of the node and the thickness of the line connecting the nodes are proportional to the number of participants. Combination refers to a combination of endurance exercise and dynamic resistance. Control refers to no exercise. ACE-I, angiotensin-converting enzyme inhibitor; $A R B$, angiotensin-2 receptor blocker; $C \mathrm{CB}$, calcium channel blocker.

(ACE-I $-7.33,95 \% \mathrm{CrI}-8.75$ to -5.91 ; ARB $-8.14,95 \% \mathrm{CrI}$ -9.62 to -6.69 ; CCB $-10.58,95 \%$ CrI -12.03 to -9.14 ; and diuretic $-8.06,95 \% \mathrm{CrI}-9.48$ to -6.64$)$ were effective in lowering baseline SBP (figure 5B).
A

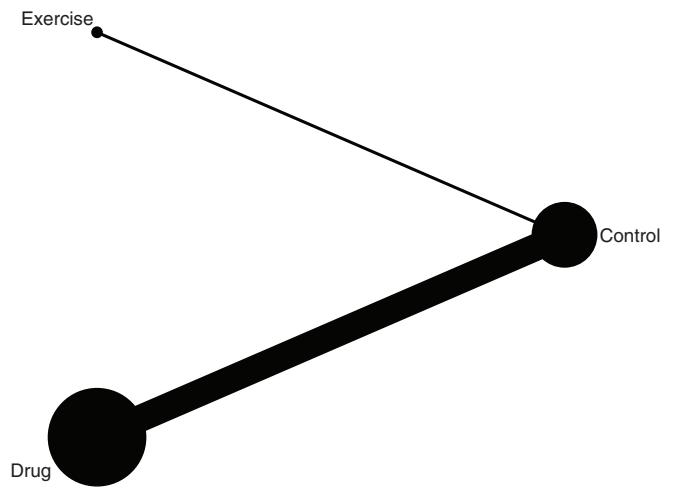

B

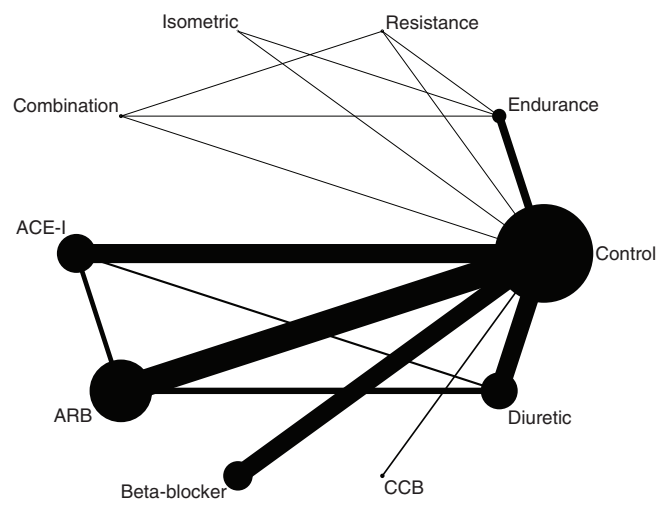

C

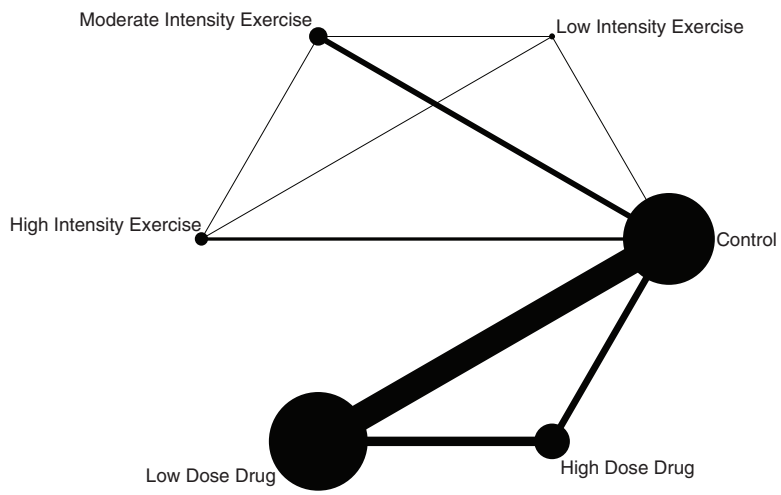

Figure 3 Available evidence in hypertensive populations comparing (A) exercise versus medications; (B) different types of exercise versus classes of medications; and (C) different intensities of exercise versus doses of medications. Combination refers to a combination of endurance exercise and dynamic resistance. Control refers to no exercise. ACE-I, angiotensin-converting enzyme inhibitor; $A R B$, angiotensin-2 receptor blocker; $C \mathrm{CB}$, calcium channel blocker.

Overall, different types of structured exercise interventions achieved similar reductions from baseline (table 2). One exception was the combination of endurance and resistance training, which was more effective in reducing baseline SBP than dynamic resistance alone $(-2.98,95 \%$ CrI -5.04 to -0.93$)$. While different classes of antihypertensive medications were generally more effective than different types of exercise interventions, 


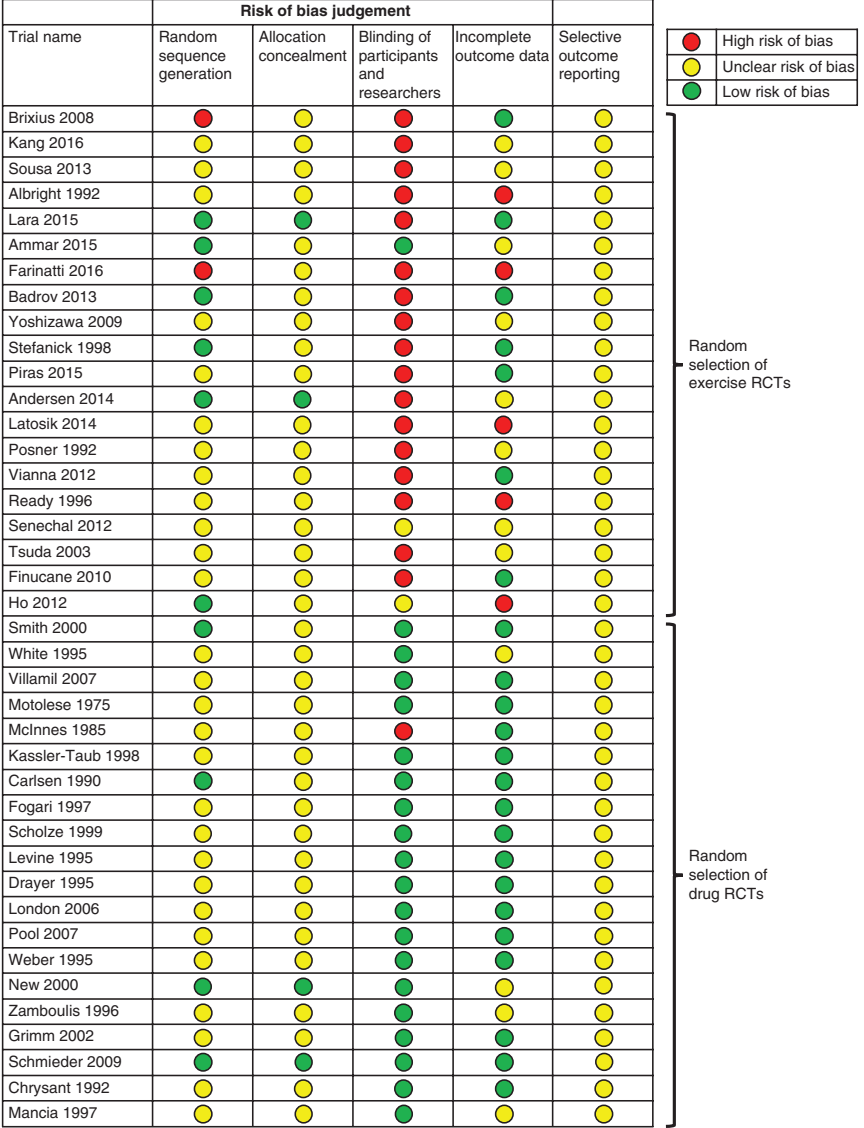

Figure 4 Risk of bias assessment of a 10\% random selection of exercise and medication randomised controlled trials.

most medication classes (ACE-I, ARB and diuretic) did not differ beyond chance from isometric resistance and combination of endurance and dynamic resistance exercises.

Participants in low- $(-4.60,95 \% \mathrm{CrI}-6.51$ to -2.69$)$, moderate- $(-5.41,95 \% \mathrm{CrI}-6.37$ to -4.46$)$ and high-intensity $(-3.87,95 \% \mathrm{CrI}-5.11$ to -2.65$)$ exercise groups achieved greater reductions in baseline SBP than those in control groups (figure 5C). Similarly, low- and high-dose medications were more effective than control, lowering baseline SBP by $8.29 \mathrm{mmHg}$ (95\% CrI -9.13 to -7.46$)$ and $10.71 \mathrm{mmHg}(95 \% \mathrm{CrI}-11.94$ to -9.46$)$, respectively. While a dose gradient was seen for medications, there was substantial uncertainty for effects of different exercise intensities.

There was no detectable evidence of inconsistency in the network meta-analyses (online supplementary appendix 6). In our small-study effects analysis, we found some evidence that smaller studies reported different results than those in larger studies for both exercise and medication interventions (online supplementary appendix 7). We observed similar model fit with both models according to total residual deviance and deviance information criterion. The estimated mean bias for exercise versus control was -1.09 (95\% CrI -1.89 to -0.34$)$ and $-1.75(95 \%$ CrI -2.61 to -0.72 ) for medications versus control; however, there was no meaningful reduction in between-study heterogeneity when we adjusted for small-study effects, suggesting that this adjustment did not necessarily explain the observed differences in effects across studies. Since the base-case model fitted well, inferences about observed improvements to model fit or lack thereof may be spurious. Regardless, models adjusted for small-study effects tended to produce smaller treatment effect

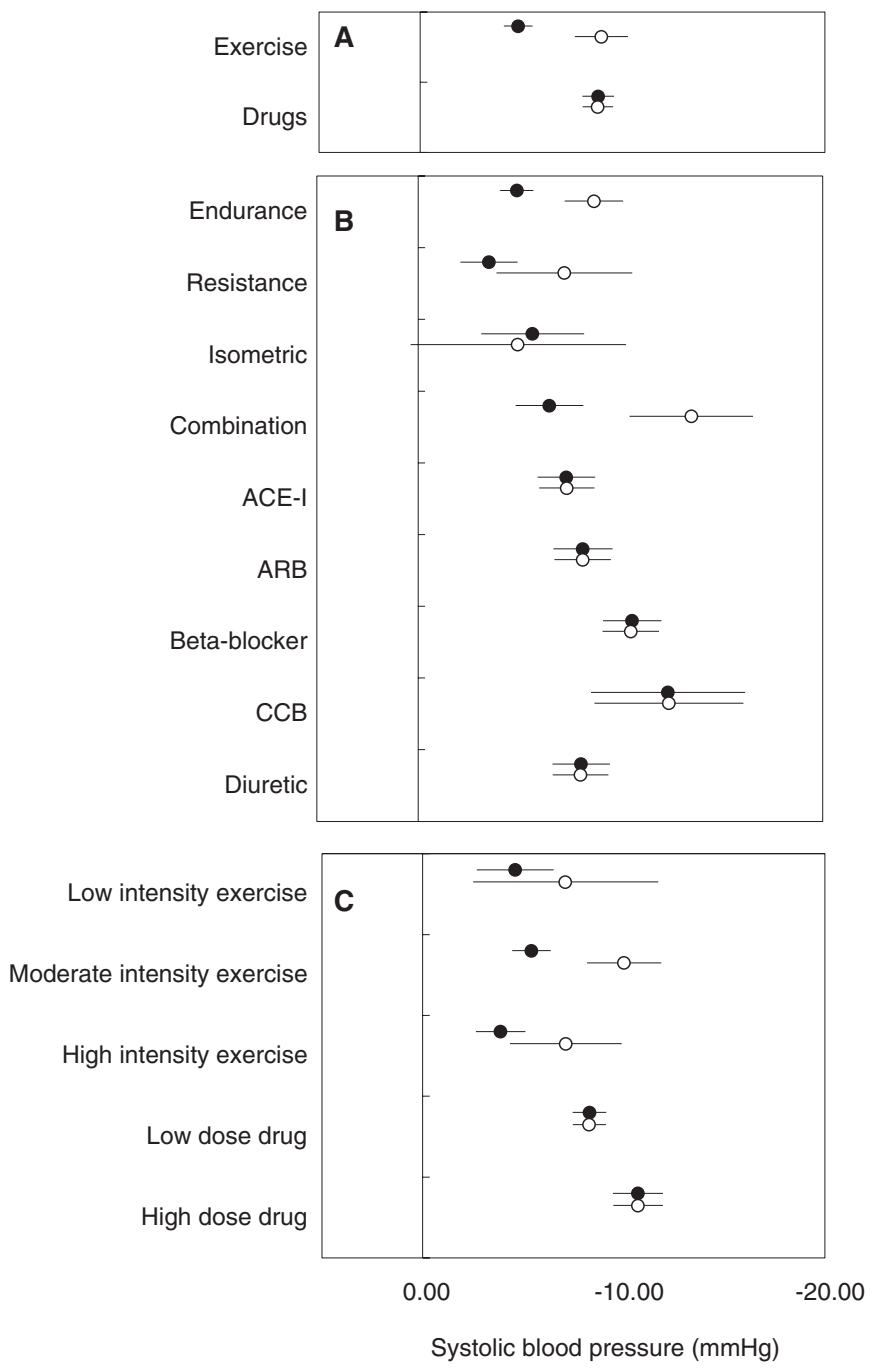

Figure 5 Findings of network meta-analyses. Change from baseline systolic blood pressure ( $\mathrm{mmHg}$ ) and 95\% Crl achieved with exercise interventions and medications as compared with control (no exercise): (A) exercise and medications; (B) different types of exercise and classes of medications; and (C) different intensities of exercise and doses of medications. Findings of analyses pooling trials from all populations are shown in black; findings of analyses restricting exercise trials to those with mean systolic blood pressure $\geq 140 \mathrm{mmHg}$ are shown in white. Combination refers to a combination of endurance exercise and dynamic training. ACE-I, angiotensin-converting enzyme inhibitor; $A R B$, angiotensin-2 receptor blocker; CCB, calcium channel blocker.

estimates for both exercise and medication interventions. Online supplementary appendix 7 compares the base-case results with predicted effect size for an ideal study of infinite size for each intervention.

\section{Comparative effects on SBP among hypertensive populations $(\geq 140 \mathrm{mmHg}$ )}

Compared with control, exercise reduced SBP by $8.96 \mathrm{mmHg}$ (95\% CrI -10.27 to -7.64$)$ among hypertensive populations $(\geq 140 \mathrm{mmHg}$ ) (figure $5 \mathrm{a}$ ). We did not observe a difference between the SBP-lowering effects of medications and exercise (0.18, $95 \%$ CrI -1.35 to 1.68$)$.

SBP was reduced (compared with control) by endurance $(-8.69,95 \% \mathrm{CrI}-10.13$ to -7.25$)$, dynamic resistance $(-7.23$, $95 \% \mathrm{CrI}-10.58$ to -3.87$)$ and their combination $(-13.51$, 


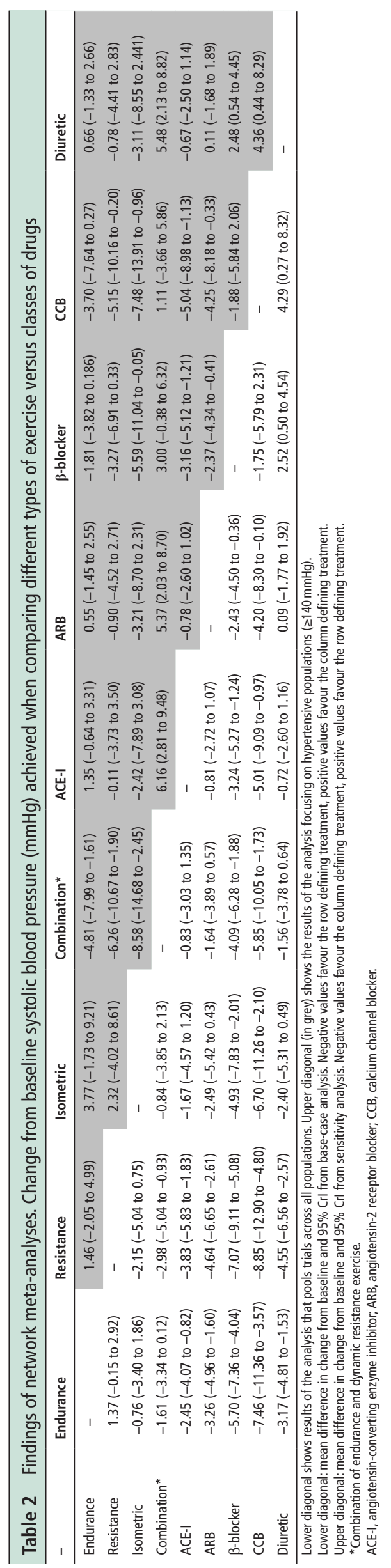

95\% CrI -16.55 to -10.45 ), while the $95 \% \mathrm{CrI}$ included the null for isometric resistance $(-4.92,95 \% \mathrm{CrI}-10.28$ to 0.38$)$ (figure 5B). Overall, different types of exercise interventions appeared similar to medications in terms of their SBP-lowering effects (table 2).

Hypertensive populations participating in moderate- and high-intensity exercise interventions achieved greater reductions in SBP compared with those in control groups (figure 5C). There were no detectable differences between different intensities of exercise and different doses of medications; however, these analyses should be interpreted with caution given the wide 95\% CrIs.

\section{Sensitivity analyses with different hypertension cut-offs}

Figure 6 and online supplementary appendix 8 show the findings of sensitivity analyses comparing the SBP-lowering effects of exercise interventions and medications at different hypertension cut-offs. Overall, exercise interventions appeared more effective as we restricted the sample of exercise trials included in the analysis to those with more hypertensive populations. For example, endurance interventions, compared with control, reduced baseline SBP by 4.88 (95\% CrI -5.69 to -4.06$)$ in the base-case analysis; respective reductions were $6.84(95 \% \mathrm{CrI}-7.90$ to -5.76$)$ in trials with $\geq 130 \mathrm{mmHg} ; 8.70$ (95\% CrI -10.13 to -7.25 ) with $\geq 140 \mathrm{mmHg}$, and 10.74 (95\% CrI -12.70 to -8.77 ) with $\geq 150 \mathrm{mmHg}$. There was substantial uncertainty in relative treatment effects when using a cut-off of $\geq 150 \mathrm{mmHg}$.

\section{DISCUSSION}

In this study, we compared the SBP-lowering effects of commonly used antihypertensive medications and exercise interventions. We found that structured exercise was often evaluated in fewer and smaller trials than medications. While the number of participants included in exercise trials accounted for approximately one third of the total in medication trials, only a 10th of the overall hypertensive population (using a $140 \mathrm{mmHg}$ cut-off) came from the exercise trials. Our analyses that synthesised the results of 391 RCTs including 39742 participants showed that individuals receiving medications achieved greater reductions in SBP than those following structured exercise regimens. However, different types of exercise interventions appeared to be as equally effective as most antihypertensive medications when we limited our analyses to trials in populations with high SBP. The effectiveness of exercise increased as we adopted higher SBP cut-offs to define hypertension.

\section{Comparison with other studies in the literature}

To the best of our knowledge, our study is the first formal evaluation of the comparative effectiveness of exercise and medications on SBP. However, a large number of previous systematic reviews and meta-analyses have examined the SBP-lowering effects of medications ${ }^{83-66}$ and exercise separately. ${ }^{27}{ }^{67-71}$ Similar to other reviews, our study identified a diverse set of exercise interventions that varied in terms of their formulation, intensity, frequency and duration. Aerobic endurance was the most frequently studied type of exercise, followed by dynamic resistance.

Our findings differed from the meta-analysis by Cornelissen and Smart in two key ways. ${ }^{27}$ First, the magnitude of SBP reduction achieved with resistance training was considerably higher in our study, likely reflecting the large numbers of newer RCTs included in our study evaluating this type of exercise and having favourable results. This was particularly so when we limited our analyses to exercise RCTs with hypertensive populations. Second, 


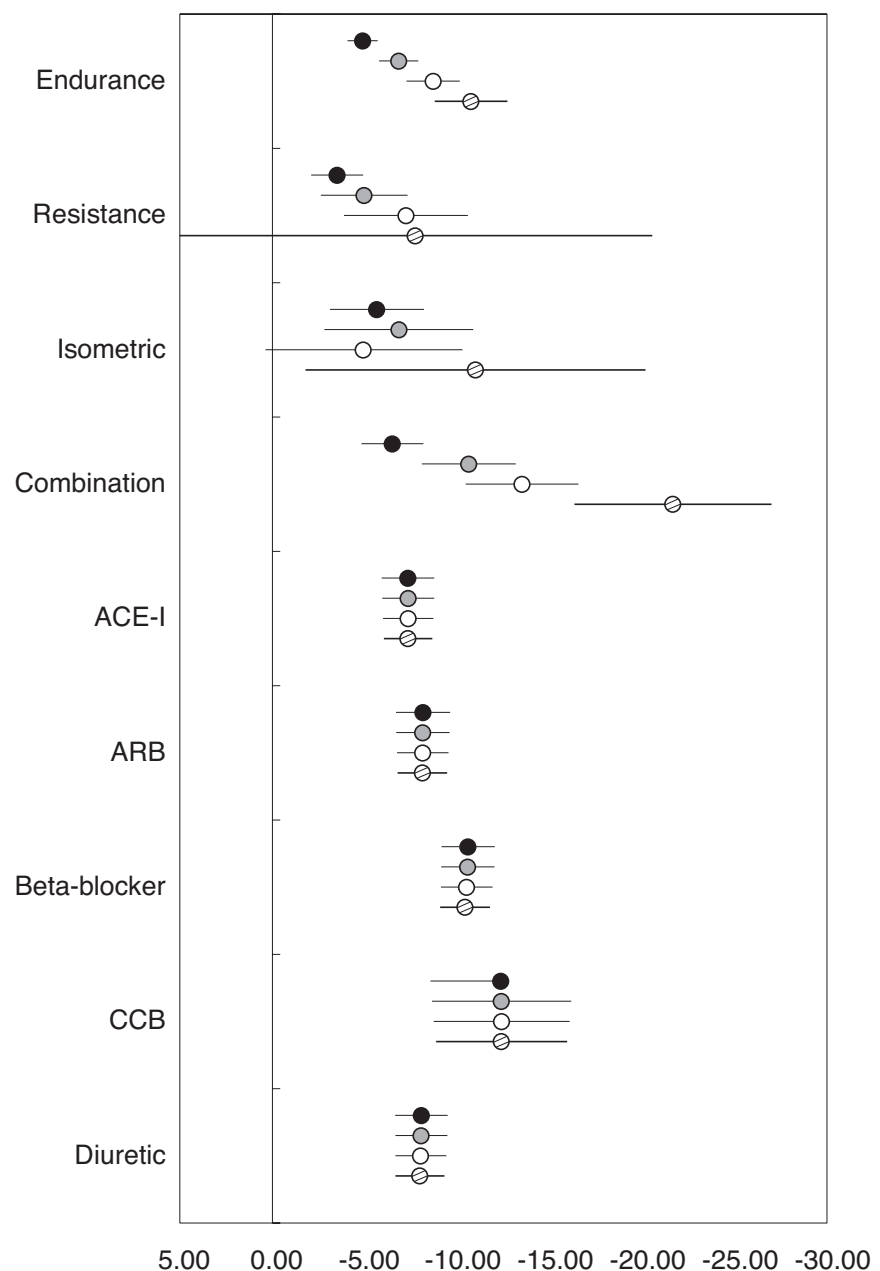

Systolic blood pressure $(\mathrm{mmHg})$

Figure 6 Findings of sensitivity network meta-analyses. Change from baseline systolic blood pressure (SBP) (mmHg) and 95\% Crl achieved with different types of exercise and drug interventions as compared with control (no exercise) using different mean SBP cut-offs. Findings of analyses pooling trials from all populations are shown in black; findings of analyses restricting exercise trials to those with mean SBP $\geq 130 \mathrm{mmHg}$ are shown in grey; $\geq 140 \mathrm{mmHg}$ are shown in white; and $\geq 150 \mathrm{mmHg}$ are shown in stripes. Combination refers to a combination of endurance exercise and dynamic training. ACE-I, angiotensinconverting enzyme inhibitor; $A R B$, angiotensin-2 receptor blocker; $C C B$, calcium channel blocker.

we found compelling evidence that combining endurance and dynamic resistance training was effective in reducing SBP. The previous review had insufficient evidence on the effectiveness of combining endurance and dynamic resistance exercise.

\section{Implications for policy and practice}

These findings could be used to examine and improve the evidence base supporting exercise recommendations. Current exercise recommendations are primarily based on observational evidence and highly variable across different settings. ${ }^{72}$ For example, in the UK, the National Health Service exercise guidelines for healthy adults 19 to 64 years of age recommend either (1) a combination of at least $150 \mathrm{~min}$ per week of moderate aerobic activity and strength training on 2 or more days a week or (2) a combination of 75 min per week of vigorous endurance activity and strength training on 2 or more days a week. ${ }^{73}$ Notably, while the guidelines specify the recommended duration of strength training, they do not specify its optimal volume and intensity. Examining and corroborating the evidence base behind these recommendations is not straightforward using the available exercise RCTs on SBP. For example, only a small subset of studies identified in our review tested the effectiveness of the combination of endurance and dynamic resistance training. Although these types of interventions were often effective, especially in hypertensive populations, their frequency and duration were not consistently reported to determine the optimal formulation and dose of physical activity to maximise its benefit. We also did not observe a dose-response relationship between exercise intensity and SBP reduction.

Recent changes to major practice guidelines developed by the AHA/ACC will substantially increase the number of people labelled as having hypertension. ${ }^{13}{ }^{14}$ While our findings support previous calls to prescribe exercise as a treatment option for hypertension ${ }^{7475}$ relatively little is known about the effectiveness and comparative effectiveness of implementation strategies for optimal exercise uptake. ${ }^{76-79}$ Healthcare systems are therefore ill-equipped to ensure effective adoption of and adherence to exercise prescriptions. Despite the availability of national guidelines promoting exercise, levels of physical activity remain stubbornly low worldwide. ${ }^{80}$ For example, an estimated $40 \%$ of adults in the Americas and many European counties are physically inactive. ${ }^{81} 82$ Given our findings that even low-intensity exercise may be effective in reducing SBP, renewed attention is warranted to identify effective strategies to promote exercise.

While our study suggests that exercise effectively lowers baseline SBP, the generalisability of these findings to real-world settings should be investigated further. Most exercise trials in our study included healthy adults with optimal or mildly elevated blood pressure. When participants had elevated SBP, exercise was often evaluated as an add-on to background antihypertensive therapy. Unlike trial populations in our review, the majority of individuals in actual clinical practice who are eligible for antihypertensive therapy have multiple chronic conditions and receive several medications. Substituting medication therapy with exercise prescriptions in these populations may therefore be challenging. ${ }^{83}$ The proportion of such patient populations who can adopt some meaningful amount of exercise is unknown. This is further compounded by the reporting quality of exercise trials. According to a recent analysis, descriptions of exercise interventions in hypertension trials were inadequately detailed for their replication in practice. ${ }^{84}$ Nevertheless, our findings can form the basis of evidence-based discussions between patients and their doctors about the SBP-lowering benefits of exercise. Although the effect of exercise is modest among individuals with moderately elevated SBP, providers should still have such discussions with their patients who are newly eligible for antihypertensive therapy as a result of recent changes to the AHA/ACC guidelines.

\section{Implications for research}

Studies comparing the effectiveness of medications and non-medication interventions are rare. Similar to our previous study ${ }^{28}$ we did not identify any RCTs that directly compared the SBP-lowering effects of antihypertensive medications and structured training programmes. To address this gap, future studies should adopt exercise as an active comparator in trials of interventions aimed at preventing, managing or treating cardiovascular diseases. Pharmaceutical companies, which in recent years 
have sponsored the majority of clinical studies, currently have little incentive to design studies that compare their products to non-medication alternatives. ${ }^{85}$ Encouraging sponsors to include established non-pharmacological alternatives in head-to-head trials will require buy-in from drug licensing agencies such as the US Food and Drug Administration and the European Medicines Agency, health technology assessment bodies such as NICE, research funders such as the National Institutes of Health in the USA and National Institute for Health Research in the UK, and research ethics committees.

In the absence of head-to-head RCTs that directly compare exercise and drug interventions, network meta-analyses can rely on existing studies and generate valuable evidence to inform policy and practice. ${ }^{86}$ Network meta-analyses that compare the benefits and harms of multiple interventions are increasingly common in the medical literature. ${ }^{8788}$ However, most consider pharmacological or non-pharmacological interventions separately. Combining these bodies of literature in network meta-analyses could identify areas where exercise interventions should be considered as viable alternatives to medications. In areas where evidence is inconclusive or biased, network meta-analyses could help inform the design of future head-to-head RCTs. ${ }^{89}$

\section{Limitations}

This study had several limitations. First, our electronic searches were conducted in Medline and did not cover other relevant bibliographic databases. Therefore, we may have missed relevant RCTs of exercise interventions if they were published in journals not indexed in Medline. Still, our review covered more than twice as many RCTs as those included in the most comprehensive systematic review to date.

Second, we did not update the list of studies included in the previously published meta-analyses of antihypertensive medications, which were published from 2008 to 2016. However, it is unlikely that we missed additional RCTs of medications, as the majority of antihypertensive medications are off patent and there is no longer an active research agenda evaluating their SBP-lowering effects.

Third, we relied on indirect evidence to determine the comparative SBP-lowering effects of exercise and medications. Such indirect comparisons could be biased if there is an imbalance in the distribution of unmeasured or unknown relative treatment effect modifiers across trials comparing different interventions. ${ }^{90}$ We summarised results from substantially heterogeneous RCTs from different settings and time periods. Trials included diverse participant populations, intervention definitions, comparators and outcome measurements. Findings of our network meta-analyses should hence be interpreted with caution. ${ }^{91}$ Transitivity may be tenuous given the typically different levels of SBP in medication versus exercise trials. However, when we tried to match trial populations more closely, the gap between medication and exercise in efficacy diminished. As we limited our analyses to more hypertensive populations, exercise interventions appeared more effective.

Fourth, while we obtained clinically relevant relative treatment effects for antihypertensive medications, we may have underestimated the magnitude of SBP lowering associated with exercise interventions. We excluded trials of medications and dosages that were considered to be ineffective or suboptimal by the BNF, even though we did not exclude any exercise trials on this basis, as there is no equivalent body that is responsible for evaluating the effectiveness of structured exercise interventions and determining their suitability as treatment options in clinical practice.
Fifth, our review shares the limitations of the studies on which it is based. Although we did not formally evaluate the internal validity of all included RCTs, we conducted a risk of bias assessment in a $10 \%$ random sample. Both exercise and medication trials had limitations in their reporting, which severely limited our accurate evaluation. We considered exercise trials to be at higher risk of performance and detection bias as compared with medication trials, primarily due to lack of blinding of investigators and participants in exercise trials. This may have greater implications on the reliability of estimates from exercise trials given the semi-objective nature of SBP lowering. However, a recent meta-epidemiological study found no significant association between treatment effects and adequate blinding in physical therapy trials. ${ }^{92}$ In contrast to the findings of a recent comprehensive review that showed a different level of reporting bias between meta-analyses of pharmacological and non-pharmacological interventions, ${ }^{93}$ we observed a similar relationship between trial size and magnitude of effect for both exercise and medications. Small-study effects may reflect publication bias, differential presence of quality issues in smaller trials, but also many other factors. ${ }^{94}$ Although exercise trials tended to be smaller, antihypertensive medication trials were not immune to small-study effects, which could explain our findings.

Exercise trials had several other methodological drawbacks. The majority of exercise trials included healthy adults with optimal blood pressure at baseline; reduction in blood pressure may be larger when the starting point is higher. Also, unlike antihypertensive medication trials, most exercise trials evaluated SBP only as secondary or tertiary outcomes; sample sizes were often too small to adequately control for confounding and produce reliable estimates of treatment effects; the composition of control arms was heterogeneous (eg, some trials included exercise as an add-on to background antihypertensive therapy while others did not allow for such therapy); and the methods used to measure SBP varied considerably across trials (eg, ranging from office-based measurement to ambulatory measurement). Similar to the findings of a recent analysis, reporting of harms associated with exercise interventions was rare and inconsistent. ${ }^{84}$ Taken together, these variations highlight the need for a more standardised approach to the design, conduct, analysis and reporting of exercise trials. Whether the reliability of estimates from exercise trials is similar to those from trials of antihypertensive medications should be evaluated in the future.

\section{CONCLUSIONS}

The effect of exercise interventions on SBP remains understudied in relation to commonly used medications, especially among hypertensive populations. Many studied types and intensities of exercise interventions demonstrate modest but consistent reductions in SBP across diverse populations and settings. The SBP-lowering effects of exercise among hypertensive populations appear similar to that of commonly used antihypertensive medications, but this is tempered by the observed differences in study population characteristics.

\section{Author affiliations}

${ }^{1}$ Department of Health Policy, London School of Economics and Political Science, London, UK

${ }^{2}$ Bristol Medical School, University of Bristol, Bristol, UK

${ }^{3}$ Centre for Reviews and Dissemination, University of York, York, UK

${ }^{4}$ Department of General Internal Medicine, Inselspital, Bern University Hospital, University of Bern, Bern, Switzerland

${ }^{5}$ Department of Health Research and Policy, Stanford University School of Medicine, Stanford, California, USA

${ }^{6}$ Meta-Research Innovation Center at Stanford (METRICS), Stanford University, Stanford, California, USA 
${ }^{7}$ Department of Life Sciences and Management, The Wharton School, University of Pennsylvania, Philadelphia, Pennsylvania, USA

${ }^{8}$ Nuffield Department of Primary Care Health Sciences, University of Oxford, Oxford, UK

${ }^{9}$ Stanford Prevention Center, Department of Medicine, Stanford University School of Medicine, Stanford, California, USA

Contributors HN and JPAI devised the paper. HN, MS-K, MRB, SAS identified studies and collected data. HN did all statistical analyses with input from SD and JPAI. HN developed the first draft of the paper and all authors contributed to subsequent versions. HN is the guarantor.

Funding HN is supported by the Higher Education Funding Council for England. SD is supported by the Medical Research Council, UK (MR/M005232/1). MRB is supported by a grant from the Swiss National Science Foundation (P2BEP3 175289).

Competing interests None declared.

Patient consent Not required.

Provenance and peer review Commissioned; externally peer reviewed.

Data sharing statement Dataset is available from the authors upon request.

\section{REFERENCES}

1 Lewington S, Clarke R, Qizilbash N, et al. Age-specific relevance of usual blood pressure to vascular mortality: a meta-analysis of individual data for one million adults in 61 prospective studies. Lancet 2002;360:1903-13.

2 Stamler J, Stamler R, Neaton JD. Blood pressure, systolic and diastolic, and cardiovascular risks. US population data. Arch Intern Med 1993;153:598-615.

3 Rutan GH, Kuller LH, Neaton JD, et al. Mortality associated with diastolic hypertension and isolated systolic hypertension among men screened for the Multiple Risk Factor Intervention Trial. Circulation 1988:77:504-14.

4 Sesso HD, Stampfer MJ, Rosner B, et al. Systolic and diastolic blood pressure, pulse pressure, and mean arterial pressure as predictors of cardiovascular disease risk in men. Hypertension 2000;36:801-7.

5 Lim SS, Vos T, Flaxman AD, et al. A comparative risk assessment of burden of disease and injury attributable to 67 risk factors and risk factor clusters in 21 regions, 19902010: a systematic analysis for the Global Burden of Disease Study 2010. Lancet 2012;380:2224-60

6 Moser M. Evolution of the treatment of hypertension from the 1940s to JNC V. Am J Hypertens 1997;10:2S-8.

7 Karmali KN, Lloyd-Jones DM, Berendsen MA, et al. Drugs for primary prevention of atherosclerotic cardiovascular disease: an overview of systematic reviews. JAMA Cardiol 2016;1:341-9.

8 Blood Pressure Lowering Treatment Trialists' Collaboration. Blood pressure-lowering treatment based on cardiovascular risk: a meta-analysis of individual patient data. Lancet 2014:384:591-8.

9 Moran AE, Forouzanfar MH, Roth GA, et al. The global burden of ischemic heart disease in 1990 and 2010: the Global Burden of Disease 2010 study. Circulation 2014;129:1493-501.

$10 \mathrm{Gu}$ Q, Burt VL, Dillon CF, et al. Trends in antihypertensive medication use and blood pressure control among United States adults with hypertension: the National Health And Nutrition Examination Survey, 2001 to 2010. Circulation 2012;126:2105-14.

11 NHS Digital. Prescriptions Dispensed in the Community England 2006 to 2016. 2017 https://files.digital.nhs.uk/publication/s/o/pres-disp-com-eng-2006-16-rep.pdf.

12 Whelton PK, Carey RM, Aronow WS, et al. 2017 ACC/AHA/AAPA/ABC/ACPM/AGS/ APhA/ASH/ASPC/NMA/PCNA guideline for the prevention, detection, evaluation, and management of high blood pressure in sdults: Executive Summary: a report of the American College of Cardiology/American Heart Association Task Force on Clinical Practice Guidelines. Hypertension 2018:71:1269-324.

13 Khera R, Lu Y, Lu J, et al. Impact of 2017 ACC/AHA guidelines on prevalence of hypertension and eligibility for antihypertensive treatment in United States and China: nationally representative cross sectional study. BMJ 2018;362:k2357.

14 Muntner P, Carey RM, Gidding S, et al. Potential US population impact of the 2017 ACC/AHA high blood pressure guideline. Circulation 2018;137:109-18.

15 Kantor ED, Rehm CD, Haas JS, et al. Trends in prescription drug use among adults in the United States from 1999-2012. JAMA 2015:314:1818-31.

16 Hajjar ER, Cafiero AC, Hanlon JT. Polypharmacy in elderly patients. Am J Geriatr Pharmacother 2007:5:345-51.

17 OECD. Health at a Glance. 2017 https://www.oecd-ilibrary.org/social-issuesmigration-health/health-at-a-glance 19991312.

18 Kesselheim AS, Avorn J, Sarpatwari A. The high cost of prescription drugs in the United States: origins and prospects for reform. JAMA 2016;316:858-71.

19 Berlin JA, Colditz GA. A meta-analysis of physical activity in the prevention of coronary heart disease. Am J Epidemiol 1990;132:612-28.

20 Warburton DE, Nicol CW, Bredin SS. Health benefits of physical activity: the evidence. CMAJ 2006;174:801-9.

21 Li J, Siegrist J. Physical activity and risk of cardiovascular disease-a meta-analysis of prospective cohort studies. Int J Environ Res Public Health 2012;9:391-407.
22 Murtagh EM, Nichols L, Mohammed MA, et al. The effect of walking on risk factors for cardiovascular disease: an updated systematic review and meta-analysis of randomised control trials. Prev Med 2015;72:34-43.

23 Chudyk A, Petrella RJ. Effects of exercise on cardiovascular risk factors in type 2 diabetes: a meta-analysis. Diabetes Care 2011;34:1228-37.

24 Kelley GA, Kelley KS, Franklin B. Aerobic exercise and lipids and lipoproteins in patients with cardiovascular disease: a meta-analysis of randomized controlled trials. J Cardiopulm Rehabil 2006:26:131-9. quiz 140-141, discussion 142-144.

25 Kodama S, Tanaka S, Saito K, et al. Effect of aerobic exercise training on serum levels of high-density lipoprotein cholesterol: a meta-analysis. Arch Intern Med 2007:167:999-1008

26 Whelton SP, Chin A, Xin X, et al. Effect of aerobic exercise on blood pressure: a metaanalysis of randomized, controlled trials. Ann Intern Med 2002:136:493-503.

27 Cornelissen VA, Smart NA. Exercise training for blood pressure: a systematic review and meta-analysis. J Am Heart Assoc 2013;2:e004473.

$28 \mathrm{Naci} H$, loannidis JP. Comparative effectiveness of exercise and drug interventions on mortality outcomes: metaepidemiological study. BMJ 2013:347:f5577.

29 National Institute for Health and Care Excellence. CG 127: Hypertension in adults: diagnosis and management. 2016 https://www.nice.org.uk/quidance/cg127.

30 ESH/ESC Task Force for the Management of Arterial Hypertension. 2013 Practice guidelines for the management of arterial hypertension of the European Society of Hypertension (ESH) and the European Society of Cardiology (ESC): ESH/ ESC Task Force for the Management of Arterial Hypertension. J Hypertens 2013:31:1925-38

31 Dickson J. The British National Formulary: checking, medicines and clinicians. Stud Health Technol Inform 2015:215:41-51.

32 Higgins J, Green S. Cochrane handbook for systematic reviews of interventions Version 5.1. 0: The Cochrane Collaboration, 2011

33 Caspersen CJ, Powell KE, Christenson GM. Physical activity, exercise, and physical fitness: definitions and distinctions for health-related research. Public Health Rep 1985;100:126-31.

34 Anon. American College of Sports Medicine Position Stand. The recommended quantity and quality of exercise for developing and maintaining cardiorespiratory and muscular fitness, and flexibility in healthy adults. Med Sci Sports Exerc 1998:30:975-91.

35 Higgins JPT, Altman DG, Gotzsche PC, et al. The Cochrane Collaboration's tool for assessing risk of bias in randomised trials. BMJ 2011:343:d5928.

36 Salanti G, Kavvoura FK, loannidis JP. Exploring the geometry of treatment networks. Ann Intern Med 2008;148:544-53.

37 Chaimani A, Higgins JP, Mavridis D, et al. Graphical tools for network meta-analysis in STATA. PLoS One 2013:8:e76654.

38 Caldwell DM, Ades AE, Higgins JP. Simultaneous comparison of multiple treatments: combining direct and indirect evidence. BMJ 2005;331:897-900

39 Mills EJ, Thorlund K, loannidis JP. Demystifying trial networks and network metaanalysis. BMJ 2013;346:f2914.

40 Salanti G, Higgins JP, Ades AE, et al. Evaluation of networks of randomized trials. Stat Methods Med Res 2008;17:279-301.

$41 \mathrm{Lu} \mathrm{G}$, Ades AE. Combination of direct and indirect evidence in mixed treatment comparisons. Stat Med 2004;23:3105-24.

42 Cipriani A, Higgins JP, Geddes JR, et al. Conceptual and technical challenges in network meta-analysis. Ann Intern Med 2013;159:130-7.

43 Lunn DJ, Thomas A, Best N, et al. WinBUGS-a Bayesian modelling framework: concepts, structure, and extensibility. Stat Comput 2000;10:325-37.

44 Dias S, Welton NJ, Sutton AJ, et al. NICE DSU technical support document 2: a generalised linear modelling framework for pairwise and network meta-analysis of randomised controlled trials. London: National Institute for Health and Care Excellence (NICE), 2011

45 Dias S, Sutton AJ, Ades AE, et al. Evidence synthesis for decision making 2: a generalized linear modeling framework for pairwise and network meta-analysis of randomized controlled trials. Med Decis Making 2013;33:607-17.

46 Dias S, Welton NJ, Sutton AJ, et al. Evidence synthesis for decision making 1: introduction. Med Decis Making 2013;33:597-606.

47 Borenstein M, Hedges LV, Higgins JP, et al. A basic introduction to fixed-effect and random-effects models for meta-analysis. Res Synth Methods 2010;1:97-111.

48 Dias S, Welton NJ, Sutton AJ, et al. Inconsistency in networks of evidence based on randomised controlled trials. London: NICE Decision Support Unit, 2011. NICE DSU Technical Support Document 4

49 Dias S, Welton NJ, Sutton AJ, et al. Evidence synthesis for decision making 4 inconsistency in networks of evidence based on randomized controlled trials. Med Decis Making 2013:33:641-56.

$50 \mathrm{Lu} \mathrm{G}$, Ades AE. Assessing evidence inconsistency in mixed treatment comparisons. J Am Stat Assoc 2006:101:447-59.

51 Dias S, Sutton AJ, Welton NJ, et al. Heterogeneity: subgroups, meta-regression, bias and bias-adjustment. London: National Institute for Health and Care Excellence (NICE), 2011

52 Dias S, Sutton AJ, Welton NJ, et al. Evidence synthesis for decision making 3: heterogeneity-subgroups, meta-regression, bias, and bias-adjustment. Med Decis Making 2013;33:618-40. 
53 Moreno SG, Sutton AJ, Turner EH, et al. Novel methods to deal with publication biases: secondary analysis of antidepressant trials in the FDA trial registry database and related journal publications. BMJ 2009;339:b2981.

54 Moreno SG, Sutton AJ, Ades AE, et al. Assessment of regression-based methods to adjust for publication bias through a comprehensive simulation study. BMC Med Res Methodol 2009;9:2.

55 Dias S, Welton NJ, Marinho VCC, et al. Estimation and adjustment of bias in randomized evidence by using mixed treatment comparison meta-analysis. J $R$ Stat Soc Ser A Stat Soc 2010;173:613-29

56 Heran BS, Wong MM, Heran IK, et al. Blood pressure lowering efficacy of angiotensin receptor blockers for primary hypertension. Cochrane Database Syst Rev 2008:CD003822.

57 Heran BS, Wong MM, Heran IK, et al. Blood pressure lowering efficacy of angiotensin converting enzyme (ACE) inhibitors for primary hypertension. Cochrane Database Syst Rev 2008:CD003823.

58 Ghamami N, Chiang SH, Dormuth C, et al. Time course for blood pressure lowering of dihydropyridine calcium channel blockers. Cochrane Database Syst Rev 2014;8:CD010052

59 Musini VM, Nazer M, Bassett K, et al. Blood pressure-lowering efficacy of monotherapy with thiazide diuretics for primary hypertension. Cochrane Database Syst Rev 2014:CD003824.

60 Wong GW, Boyda HN, Wright JM. Blood pressure lowering efficacy of partial agonist beta blocker monotherapy for primary hypertension. Cochrane Database Syst Rev 2014:CD007450.

61 Wong GW, Boyda HN, Wright JM. Blood pressure lowering efficacy of beta-1 selective beta blockers for primary hypertension. Cochrane Database Syst Rev 2016:3:CD007451

62 Wong GW, Wright JM. Blood pressure lowering efficacy of nonselective beta-blockers for primary hypertension. Cochrane Database Syst Rev 2014:CD007452.

63 Blood Pressure Lowering Treatment Trialists'Collaboration. Effects of ACE inhibitors, calcium antagonists, and other blood-pressure-lowering drugs: results of prospectively designed overviews of randomised trials. The Lancet 2000;356:1955-64.

64 Turnbull F. Blood Pressure Lowering Treatment Trialists' Collaboration. Effects of different blood-pressure-lowering regimens on major cardiovascular events: results of prospectively-designed overviews of randomised trials. Lancet 2003;362:1527-35.

65 Turnbull F, Neal B, Ninomiya T, et al. Effects of different regimens to lower blood pressure on major cardiovascular events in older and younger adults: meta-analysis of randomised trials. BMJ 2008:336:1121-3.

66 Ettehad D, Emdin CA, Kiran A, et al. Blood pressure lowering for prevention of cardiovascular disease and death: a systematic review and meta-analysis. Lancet 2016:387:957-67.

67 Cornelissen VA, Fagard RH. Effect of resistance training on resting blood pressure: a meta-analysis of randomized controlled trials. J Hypertens 2005;23:251-9.

68 Cornelissen VA, Fagard RH. Effects of endurance training on blood pressure, blood pressure-regulating mechanisms, and cardiovascular risk factors. Hypertension 2005;46:667-75.

69 Hanson S, Jones A. Is there evidence that walking groups have health benefits? A systematic review and meta-analysis. Br J Sports Med 2015;49:710-5.

70 Inder JD, Carlson DJ, Dieberg G, et al. Isometric exercise training for blood pressure management: a systematic review and meta-analysis to optimize benefit. Hypertens Res 2016;39:88-94.

71 Casonatto J, Goessler KF, Cornelissen VA, et al. The blood pressure-lowering effect of a single bout of resistance exercise: a systematic review and meta-analysis of randomised controlled trials. Eur J Prev Cardiol 2016;23:1700-14.
72 Kahlmeier S, Wijnhoven TM, Alpiger P, et al. National physical activity recommendations: systematic overview and analysis of the situation in European countries. BMC Public Health 2015;15:133.

73 NHS Choices. Physical activity guidelines for adults. https://www.nhs.uk/live-well/ exercise/.

74 Pedersen BK, Saltin B. Evidence for prescribing exercise as therapy in chronic disease. Scand J Med Sci Sports 2006;16(Suppl 1):3-63.

75 Pedersen BK, Saltin B. Exercise as medicine - evidence for prescribing exercise as therapy in 26 different chronic diseases. Scand J Med Sci Sports 2015;25:1-72.

76 Conn VS, Hafdahl AR, Mehr DR. Interventions to increase physical activity among healthy adults: meta-analysis of outcomes. Am J Public Health 2011;101:751-8.

77 Williams NH, Hendry M, France B, et al. Effectiveness of exercise-referral schemes to promote physical activity in adults: systematic review. $\mathrm{Br} J$ Gen Pract 2007; 57:979-86

78 Pavey TG, Taylor AH, Fox KR, et al. Effect of exercise referral schemes in primary care on physical activity and improving health outcomes: systematic review and metaanalysis. BMJ 2011;343:d6462.

79 Orrow G, Kinmonth AL, Sanderson S, et al. Effectiveness of physical activity promotion based in primary care: systematic review and meta-analysis of randomised controlled trials. BMJ 2012;344:e1389.

80 Kohl HW, Craig CL, Lambert EV, et al. The pandemic of physical inactivity: global action for public health. Lancet 2012;380:294-305.

81 Hallal PC, Andersen LB, Bull FC, et al. Global physical activity levels: surveillance progress, pitfalls, and prospects. Lancet 2012;380:247-57.

82 European Commission. Special Eurobarometer 412 - sport and physical activity. 2014 http://ec.europa.eu/commfrontoffice/publicopinion/archives/ebs/ebs_412_en.pdf.

83 loannidis JPA. Diagnosis and treatment of hypertension in the 2017 ACC/AHA guidelines and in the real world. JAMA 2018;319:115-6.

84 Hacke C, Nunan D, Weisser B. Do exercise trials for hypertension adequately report interventions? A reporting quality study. Int J Sports Med 2018;39:902-8.

85 Lathyris DN, Patsopoulos NA, Salanti G, et al. Industry sponsorship and selection of comparators in randomized clinical trials. Eur J Clin Invest 2010;40:172-82.

86 Ioannidis J. Next-generation systematic reviews: prospective meta-analysis, individuallevel data, networks and umbrella reviews. Br J Sports Med 2017;51:1456-8.

87 Zarin W, Veroniki AA, Nincic V, et al. Characteristics and knowledge synthesis approach for 456 network meta-analyses: a scoping review. BMC Med 2017;15:3.

88 Nikolakopoulou A, Chaimani A, Veroniki AA, et al. Characteristics of networks of interventions: a description of a database of 186 published networks. PLoS One 2014;9:e86754.

89 Salanti G, Nikolakopoulou A, Sutton AJ, et al. Planning a future randomized clinical trial based on a network of relevant past trials. Trials 2018;19:365.

90 Jansen JP, Naci H. Is network meta-analysis as valid as standard pairwise meta-analysis? It all depends on the distribution of effect modifiers. BMC Med 2013;11:159.

91 Mills EJ, loannidis JP, Thorlund K, et al. How to use an article reporting a multiple treatment comparison meta-analysis. JAMA 2012;308:1246-53.

92 Armijo-Olivo S, Fuentes J, da Costa BR, et al. Blinding in physical therapy trials and its association with treatment effects: a meta-epidemiological study. Am J Phys Med Rehabil 2017;96:34-4.

93 Rezende LFM, Rey-López JP, Sá TH, et al. Reporting bias in the literature on the associations of health-related behaviors and statins with cardiovascular disease and all-cause mortality. PLoS Biol 2018;16:e2005761.

94 Sterne JA, Sutton AJ, loannidis JP, et al. Recommendations for examining and interpreting funnel plot asymmetry in meta-analyses of randomised controlled trials. BMJ 2011:343:d4002. 